\title{
OPEN Assessment of metastatic lymph nodes in head and neck squamous cell carcinomas using simultaneous ${ }^{18}$ F-FDG-PET and MRI
}

\author{
Jenny Chen ${ }^{1}$, Mari Hagiwara ${ }^{1}$, Babak Givi ${ }^{2}$, Brian Schmidt ${ }^{3}$, Cheng Liu ${ }^{4}$, Qi Chen ${ }^{1}$, \\ Jean Logan ${ }^{1}$, Artem Mikheev ${ }^{1}$, Henry Rusinek ${ }^{1}$ \& Sungheon Gene Kim ${ }^{1,5 \bowtie}$
}

In this study, we investigate the feasibility of using dynamic contrast enhanced magnetic resonance imaging (DCE-MRI), diffusion weighted imaging (DWI), and dynamic positron emission tomography (PET) for detection of metastatic lymph nodes in head and neck squamous cell carcinoma (HNSCC) cases. Twenty HNSCC patients scheduled for lymph node dissection underwent DCE-MRI, dynamic PET, and DWI using a PET-MR scanner within one week prior to their planned surgery. During surgery, resected nodes were labeled to identify their nodal levels and sent for routine clinical pathology evaluation. Quantitative parameters of metastatic and normal nodes were calculated from DCE-MRI $\left(\mathrm{v}_{\mathrm{e}}, \mathrm{v}_{\mathrm{p}}, \mathrm{PS}, \mathrm{F}_{\mathrm{p}}, \mathrm{K}^{\text {trans }}\right)$, DWI (ADC) and PET $\left(\mathrm{K}_{\mathrm{i}}, \mathrm{K}_{1}, \mathrm{k}_{2}, \mathrm{k}_{3}\right)$ to assess if an individual or a combination of parameters can classify normal and metastatic lymph nodes accurately. There were 38 normal and 11 metastatic nodes covered by all three imaging methods and confirmed by pathology. $34 \%$ of all normal nodes had volumes greater than or equal to the smallest metastatic node while 4 normal nodes had SUV > 4.5. Among the MRI parameters, the median $v_{p}, F_{p}, P S$, and $K^{\text {trans }}$ values of the metastatic lymph nodes were significantly lower $(p=<0.05)$ than those of normal nodes. $v_{\mathrm{e}}$ and ADC did not show any statistical significance. For the dynamic PET parameters, the metastatic nodes had significantly higher $\mathrm{k}_{3}\left(p\right.$ value $\left.=8.8 \times 10^{-8}\right)$ and $\mathrm{K}_{\mathrm{i}}\left(p\right.$ value $\left.=5.3 \times 10^{-8}\right)$ than normal nodes. $\mathrm{K}_{1}$ and $\mathrm{k}_{2}$ did not show any statistically significant difference. $K_{i}$ had the best separation with accuracy $=0.96$ (sensitivity $=1$, specificity $=0.95$ ) using a cutoff of $\mathrm{K}_{\mathrm{i}}=5.3 \times 10^{-3} \mathrm{~mL} / \mathrm{cm}^{3} / \mathrm{min}$, while $\mathrm{k}_{3}$ and volume had accuracy of 0.94 (sensitivity $=0.82$, specificity $=0.97$ ) and 0.90 (sensitivity $=0.64$, specificity $=0.97$ ) respectively. $100 \%$ accuracy can be achieved using a multivariate logistic regression model of MRI parameters after thresholding the data with $\mathrm{K}_{\mathrm{i}}<5.3 \times 10^{-3} \mathrm{~mL} / \mathrm{cm}^{3} / \mathrm{min}$. The results of this preliminary study suggest that quantitative MRI may provide additional value in distinguishing metastatic nodes, particularly among small nodes, when used together with FDG-PET.

Head and neck cancer (HNC) represents approximately $4 \%$ of invasive cancers diagnosed annually in the United States $^{1}$. Approximately 65,000 Americans develop HNC each year and nearly 14,500 patients with HNC die from it each year. Worldwide, more than 500,000 individuals will develop HNC each year, ranking it as the sixth most common cancer. These cancers predominately originate from mutations in the squamous cells and approximately two thirds of patients will present with locally advanced disease with either large disease at the primary site and/or spread to regional lymph nodes. Approximately $50 \%$ of patients presenting with these advanced diseases survive for more than 5 years.

Accurate identification and characterization of lymph node metastasis by non-invasive imaging has important therapeutic and prognostic significance in patients with newly diagnosed HNC as well as in evaluating treatment response $^{2-5}$. It is crucial to be informed about the absence or presence of nodal metastasis before commencing any

\footnotetext{
${ }^{1}$ Department of Radiology, Center for Biomedical Imaging (CBI), Center for Advanced Imaging Innovation and Research (CAI2R), New York University School of Medicine, 660 First Avenue, New York, NY 10016, USA. ${ }^{2}$ Department of Otolaryngology-Head and Neck Surgery, New York University School of Medicine, New York, NY, USA. ${ }^{3}$ Department of Oral and Maxillofacial Surgery, Bluestone Center for Clinical Research, New York University College of Dentistry, New York, NY, USA. ${ }^{4}$ Department of Pathology, New York University School of Medicine, New York, NY, USA. ${ }^{5}$ Department of Radiology, Weill Cornell Medical College, New York, NY, USA. ${ }^{\boxplus}$ email: sgk4001@med.cornell.edu
} 
therapy. Accurate information about the presence and location of nodal metastases can prove useful in planning the appropriate nodal dissection to address all involved sites; and avoid unnecessary extensive operations with their side effects on quality of life and increasing the risk of lymphedema. While the need for a highly accurate imaging method for assessment of lymph nodes is paramount, such need is not adequately met by currently available diagnostic imaging methods ${ }^{6-8}$.

Cross-sectional imaging modalities rely on size and morphologic criteria which have limited sensitivity and specificity and thus lack the desired accuracy for characterizing lymph nodes in HNSCC. Identification of metastatic lymph nodes with magnetic resonance imaging (MRI) based on nodal size is limited as demonstrated by variable sensitivity and specificity reported depending on the size criteria used. The limitations of this sizebased characterization system are well known: metastases can be present in non-enlarged lymph nodes and not all enlarged nodes are malignant ${ }^{9}$. Identification of lymph node necrosis on conventional imaging is a reliable sign for nodal metastasis, but demonstrates a limited negative predictive value, particularly with smaller nodes. Thus, detection of lymph node metastasis based on nodal size and the presence of necrosis remains difficult.

Currently, advanced functional MRI methods, such as diffusion ${ }^{10-12}$ and perfusion ${ }^{13}$ imaging methods, have been used to detect metastatic lymph nodes. Metastasis in the lymph nodes may be associated with increased cell density leading to alterations in water diffusivity which can be measured as apparent diffusion coefficient (ADC) using diffusion weighted MRI (DWI). Sumi et al. ${ }^{10}$ reported that the metastatic lymph nodes could have a wide range of ADC in $\mathrm{HNC}$ and interestingly, the ADC values in the nodes with metastases from poorly differentiated HNC was significantly lower than that from moderately to highly differentiated HNC. However, the accuracy of ADC measurements may be limited by relatively large voxel size and low signal-to-noise ratio (SNR) ${ }^{10,12}$. Dynamic contrast enhanced (DCE)-MRI has been widely used to assess tumor vascularity. A meta-analysis of 43 papers on application of DCE-MRI for lymph node assessment found that the overall accuracy of gadoliniumbased DCE-MRI for the detection of nodal metastases is moderate $(72 \% \text { sensitivity and } 87 \% \text { specificity })^{13}$.

In contrast, positron emission tomography (PET) imaging using $18 \mathrm{~F}$-fluorodeoxyglucose $\left({ }^{18} \mathrm{~F}-\mathrm{FDG}\right)$ has been found to have high sensitivity $(92-100 \%)$ in detecting nodal metastases with mixed specificity $(77-93 \%)^{14,15}$. Investigators in previous studies have evaluated the role of FDG-PET for the detection of lymph node metastasis in patients with HNC mainly by comparing imaging findings with surgical or clinical follow-up findings. Histopathologic correlation has been available in only a few studies ${ }^{16}$. Previous studies investigating fusion techniques of PET and MR imaging found that using these two modalities could improve both sensitivity and specificity for detecting metastatic lymph nodes in $\mathrm{HNC}^{17,18}$. However, there is a limited number of studies using the hybrid PET/MRI scanners for assessment of lymph nodes in HNSCC, combined with quantitative data analysis methods for individual modalities.

Therefore, the purpose of this study is to investigate the feasibility of using dynamic FDG-PET with DCE-MRI and DWI in synergy to assess the metastatic status of lymph nodes in HNSCC patients by obtaining quantitative DCE-MRI and FDG-PET kinetic parameters. In this study, we take advantage of using a PET/MR scanner to acquire DCE-MRI, DWI, and dynamic FDG-PET data simultaneously. The hypothesis of this study is that quantitative parameters from all three imaging methods can accurately classify metastatic lymph nodes from normal ones.

\section{Methods}

Participants. Patients with biopsy-proven head and neck squamous cell carcinoma (HNSCC) ( $\mathrm{n}=20$, mean age $62 \pm 16$ years; 15 males and 5 females; Table 1) who were scheduled for cervical lymph node dissection as part of their standard treatment at NYU Langone Medical Center or Bellevue hospital were recruited for this HIPAA-compliant institutional review board-approved study. This research was performed in accordance with relevant guidelines and regulations and written informed consent was obtained from each subject. The primary cancers were squamous cell carcinomas of the oral cavity $(n=14)$, oropharynx $(n=2)$, nose $(n=1)$, larynx $(n=1)$, and unknown primary $(\mathrm{n}=2)$. Each patient had one research scan using a whole body 3T PET-MR scanner (Biograph mMR, Siemens Healthcare) at the Center for Biomedical Imaging, Department of Radiology, New York University Langone Medical Center, within one week prior to their planned surgery.

Identification of nodes. During the surgery, the resected lymph nodes were labeled to identify their nodal levels and served as a guide to locate the corresponding nodes on images ${ }^{19}$. In summary, level I nodes include submental and submandibular nodes, level II nodes are the upper jugular nodes, level III nodes are the middle jugular nodes, level IV nodes are the lower jugular nodes, level V nodes are the posterior triangle nodes, and level VI nodes are the anterior compartment and pretracheal nodes. Surgical specimens were labelled for each nodal level by the operating surgeon and sent for clinical pathology evaluation. Then, on post-contrast MRI, an experienced neuroradiologist ( $\mathrm{MH}$ with 12 years of experience) manually selected 3D regions of interest (ROI) for internal carotid artery, normal/negative $(n=38)$ and metastatic/positive $(n=11)$ nodes that were included in the fields of view of three imaging modalities (DCE-MRI, DWI, and PET) and also specified by the operative note from the neck dissection and pathology reports. The ROIs were drawn so the central necrotic region was included and in every slice where the node was visible. The number of slices per ROI varies from 6 to 25 slices for metastatic nodes and 2 to 25 slices for normal nodes.

PET data acquisition. Subjects were instructed to fast for at least $6 \mathrm{~h}$ before their scans. The PET scan started $1 \mathrm{~min}$ before injection of $10 \mathrm{mCi}{ }^{18} \mathrm{~F}$-FDG into an antecubital vein and was acquired dynamically for $60 \mathrm{~min}$ (38 timeframes; 5 frames of $10 \mathrm{s,}, 20 \mathrm{s,}, 30 \mathrm{~s}, 60 \mathrm{~s}$, and $100 \mathrm{~s}$ each, 12 frames of $200 \mathrm{~s}$, and 1 frame of $40 \mathrm{~s}$ ). Attenuation correction maps were estimated using water and fat images generated from T1-weighted gradientecho images. PET images were reconstructed using 3D ordinary Poisson ordered subset expectation maximiza- 


\begin{tabular}{|l|l|l|l|l|}
\hline Patient & Age & Gender & Tumor primary location & Stage \\
\hline 1 & 50 & M & Mandible & T2N2aM0 \\
\hline 2 & 80 & M & Base of tongue & T1N0M0 \\
\hline 3 & 70 & M & Base of tongue & T1N1M0 \\
\hline 4 & 80 & M & Nose & T0N2cM0 \\
\hline 5 & 70 & M & Tongue & T1N0M0 \\
\hline 6 & 50 & M & Buccal mucosa & T1N0M0 \\
\hline 7 & 35 & M & Tongue & T2N2bM0 \\
\hline 8 & 93 & M & Mandible & T2N0M0 \\
\hline 9 & 65 & M & Tongue & T1N0M0 \\
\hline 10 & 73 & F & Tongue & T2N2cM0 \\
\hline 11 & 45 & M & Tongue & T1N2M0 \\
\hline 12 & 55 & M & Floor of mouth & T1N0M0 \\
\hline 13 & 64 & F & Gingiva & T4N1M0 \\
\hline 14 & 27 & F & Tongue & T1N0M0 \\
\hline 15 & 72 & F & Floor of mouth & T1N0M0 \\
\hline 16 & 62 & M & Unknown primary & TxN2aM0 \\
\hline 17 & 60 & M & Mandible & T4aN2bM0 \\
\hline 18 & 73 & F & Mandible & T2N2bM0 \\
\hline 19 & 52 & M & Larynx & T4aN1M0 \\
\hline 20 & 57 & M & Unknown primary & TxN2bM0 \\
\hline
\end{tabular}

Table 1. Summary of $n=20$ patients' age, gender, and tumor type, primary location, and stage. All patients had biopsy-proven HNSCC.

tion (OP-OSEM) (127 axial slices, $344 \times 344$ matrix; $2 \times 2 \times 2 \mathrm{~mm}^{3}$ voxel dimensions ${ }^{20}$. A post-reconstruction smoothing with a Gaussian filter and kernel width of $2 \mathrm{~mm}$ full width at half maximum was applied.

MRI data acquisition. MRI data were acquired simultaneously during the PET data acquisition. The research imaging sequences described below took a total of $20 \mathrm{~min}$.

DWI was conducted using a twice-refocused spin echo sequence with echo planar readout $(\mathrm{TR}=6000 \mathrm{~ms}$, TE $64 \mathrm{~ms}$, flip angle (FA) $180^{\circ}$, matrix $128 \times 128 \times 27$, resolution $2 \times 2 \times 4.4 \mathrm{~mm}^{3}$ ) with four b-values: $0,200,500$, and $800 \mathrm{~s} / \mathrm{mm}^{2}$. The data acquisition with a non-zero b-value was repeated three times with three orthogonal diffusion weighting directions.

T1 mapping was conducted with either MP2RAGE sequence ${ }^{21}$ for five subjects and the variable flip angle (VFA) method ${ }^{22}$ for ten subjects. T1 maps were not available for the remaining five patients, so the average value from 15 subjects was used for the data analysis. MP2RAGE is a variation of standard 3D magnetization-prepared rapid gradient-echo (MPRAGE) sequence, with two inversion times (700 and $2500 \mathrm{~ms}$ ) with FA of $4^{\circ}$ and $5^{\circ}$ respectively (TR $5000 \mathrm{~ms}$, TE $2.88 \mathrm{~ms}$, 176 sagittal slices, matrix $256 \times 240$, resolution $1 \times 1 \times 1.2 \mathrm{~mm}^{3}$ ). The VFA method used five flip angles using 3D fat suppressed T1-weighted volumetric interpolated breath-hold examination (VIBE) sequence (flip angles $=2^{\circ}, 4^{\circ}, 6^{\circ}, 10^{\circ}, 15^{\circ}$, TR $10 \mathrm{~ms}$, TE $1.6 \mathrm{~ms}, 128$ axial slices, matrix $256 \times 256$; resolution $\left.1 \times 1 \times 2 \mathrm{~mm}^{3}\right)$.

DCE-MRI scans were acquired using a golden-angle radial 3D gradient echo sequence $(\mathrm{TR}=4.38 \mathrm{~ms}$, TE $2.11 \mathrm{~ms}$, FA $9^{\circ}$, matrix $256 \times 256 \times 128$, resolution $1 \times 1 \times 2 \mathrm{~mm}^{3}$ ). The baseline images were acquired for $1 \mathrm{~min}$ before contrast injection, followed by injection of gadobutrol (Gadavist, Bayer Healthcare Pharmaceuticals) with a concentration of $0.1 \mathrm{mM} / \mathrm{kg}$ body weight at the rate of $1 \mathrm{~mL} / \mathrm{s}$ into the same antecubital vein used for the ${ }^{18} \mathrm{~F}$ FDG injection and a saline flush. The scan continued for another $7 \mathrm{~min}$. The dynamic images (76 timeframes, temporal resolution of $5.5 \mathrm{~s}$ ) were reconstructed using the golden angle radial spare parallel (GRASP) method $^{23}$.

Image registration. Images from the neck are subject to voluntary and involuntary motion such as swallowing and breathing. In order to minimize such motion artifact, image coregistration across and within modalities was conducted for each subject. In DCE-MRI, the 3D image in the middle timeframe of the scan was empirically selected as the reference image to which individual 3D images of other timeframes were co-registered individually. Co-registration was performed using SimpleElastix software (https//simpleelastix.github. io). We used a multi-resolution registration (2 resolutions) approach with b-spline interpolation (1st order in each resolution, 3 rd order in final deformation), advanced Mattes mutual information similarity metric (32 histogram bins), advanced stochastic gradient descent optimizer (4000 max iterations), and b-spline transform (10 mm minimum grid spacing $)^{24,25}$. $\mathrm{T}_{1}$ maps were registered to the reference DCE-MRI frame using SimpleITK software ${ }^{26-28}$ with a rigid transform model to match image matrix, voxel dimensions, and orientation. DWI images were first processed using FSL's TopUp tool to correct susceptibility induced distortions ${ }^{29,30}$ and the Eddy correction tool $^{31}$. After that, DWI volumes were registered to the reference DCE-MRI frame using SimpleITK with mutual information similarity metric, regular step gradient descent optimization, and b-spline transform 
and interpolation. PET images were resampled to match the DCE-MRI reference frame using FireVoxel (https ://wp.nyu.edu/firevoxel).

Data analysis. After image registration, the dynamic PET, DCE-MRI and DWI data were analyzed using FireVoxel to estimate quantitative parameters related to glucose metabolic rate, vascularity, and cellularity of the selected ROIs.

For DCE-MRI, contrast kinetic model analysis was conducted with the population-based arterial input function (AIF) model proposed by Parker et al. ${ }^{32}$. This was done in view of well-known limitations of individual image-based AIFs ${ }^{33}$. The AIF model was assumed to be the contrast concentration in blood, $\mathrm{C}_{b}(t)$. $\mathrm{C}_{b}(t)$ was shifted to match the arrival time of the signal enhancement measured in one of the internal carotid arteries by matching the population AIF peak to the internal carotid artery averaged time activity curve peak. $\mathrm{C}_{\mathrm{b}}(\mathrm{t})$ was converted to the contrast concentration in plasma, $\mathrm{C}_{\mathrm{p}}=\mathrm{C}_{\mathrm{b}} /(1-\mathrm{Hct})$, where Hct is hematocrit assumed to be $0.42^{34}$. The mean signal intensity data of a node, $\mathrm{S}(\mathrm{t})$, was converted to concentration values $\mathrm{C}_{\mathrm{t}}(\mathrm{t})$ using a linear conversion ${ }^{35}$,

$$
C_{t}(t)=\frac{1}{r_{1} T_{1}}\left(\frac{S(t)}{S(0)}-1\right)
$$

where $S(0)$ is the baseline signal, and $r_{1}$ is the contrast agent relaxivity that was assumed to be $4.5 \mathrm{mM}^{-1} \mathrm{~s}^{-1}$ here. The linear conversion was used to minimize the uncertainty that could be influenced by the five cases with an assumed $\mathrm{T} 1$ value $^{36}$. The two-compartment exchange model (2CXM) was used to estimate mean plasma volume fraction $\left(\mathrm{v}_{\mathrm{p}}\right)$, interstitial volume fraction $\left(\mathrm{v}_{\mathrm{e}}\right)$, permeability-surface area product (PS), and plasma flow $\left(\mathrm{F}_{\mathrm{p}}\right)^{37}$. Volume transfer constant $\left(\mathrm{K}^{\text {trans }}\right)$ was then calculated from the estimated $\mathrm{F}_{\mathrm{p}}$ and PS values; $\mathrm{K}^{\text {trans }}=\mathrm{F}_{\mathrm{p}}(1-\exp (-\mathrm{PS} /$ $\left.\mathrm{F}_{\mathrm{p}}\right)$ ).

The DWI data were used to estimate ADC parametric maps by a monoexponential fit to the signal intensities: $\ln (S)=\ln \left(S_{0}\right)-b^{\star} A D C$, where $S$ is the voxel signal, $b$ is the $b$-value, and $S_{0}$ is the corresponding voxel signal without vascular contribution at $b=0$. DWI data with $b=0$ was used for the image registration, but not included in the calculation of ADC such that only the data with $b \geq 200 \mathrm{~s} / \mathrm{mm}^{2}$ were used to minimize the intravoxel incoherent motion effect. We calculated the mean ADC of each lymph node ROI from the estimated ADC maps. DWI field-of-view does not cover every lymph nodes observed in other imaging modalities. Since this paper is investigating assessment of lymph nodes using a combination of DCE-MRI, DWI, and PET parameters, lymph nodes not in DWI field-of-view were excluded because these nodes would be missing diffusion parameter.

The dynamic PET data were analyzed using the irreversible two-compartment model with three parameters; ${ }^{18} \mathrm{~F}-\mathrm{FDG}$ transport rate constant from blood to tissue $\left(\mathrm{K}_{1}\right)$, transport rate constant from tissue to blood $\left(\mathrm{k}_{2}\right)$, and phosphorylation rate constant $\left(\mathrm{k}_{3}\right)^{38}$. This model was the basis of the Sokoloff method used for the estimation of metabolic rate. An image-based AIF was generated for each subject using the previously mentioned neuroradiologist manually created 3D ROIs of the internal carotid artery. A principal component analysis (PCA) method ${ }^{39}$ was used to minimize the partial volume and noise effect in many voxels within the selected ROI for the AIF. As for the input to the PCA analysis, 100 voxels from the ROI with the greatest initial area under the curve (IAUC) for the first $3 \mathrm{~min}$ of its dynamic curve were selected. Then, AIF was estimated using the major principal components that make up equal to or more than $95 \%$ of the total variance. FireVoxel, our inhouse software was used for parameter estimation of mean $K_{1}, k_{2}, k_{3}$ and the influx constant $K_{i}\left(K_{i}=K_{1} k_{3} /\left(k_{2}+k_{3}\right)\right.$ related to the metabolic rate.

Statistical data analysis. We measured mean values of the parameters in each ROI. Once quantitative parameters were estimated, comparisons between normal and metastatic lymph nodes, in terms of DCE-MRI, PET, and DWI parameters, were performed by comparing the median values of the mean ROI parameters between the groups, mainly due to a small number of samples in each group, using the Mann-Whitney $U$ test. This test was computed using SciPy, a free and open-source Python library (https://scipy.org/scipylib). The twosided 5\% significance level was used on all statistical tests. Parameter values reported below are median values with the inter-quartile ranges (IQR) in parentheses unless specified otherwise.

Using logistic regression model from Scikit-learn $(\mathrm{C}=1 \mathrm{e}$, penalty $=\mathrm{L} 2$, solver $=$ liblinear $)$, a machine learning library in Python (https://scikit-learn.org/stable/index.html), the PET, DCE-MRI, and DWI parameters along with lymph node volume were fitted to the model individually. The predicted output accuracy was used to rank the parameter or parameters by which yield the highest accuracy. The logistic function was defined as $p(x)=\frac{1}{1+e^{-f(x)}}$, where $f(x)$ is the linear function, $f(x)=b_{0}+b_{1} x+\cdots+b_{r} x_{r}$ with variables $b_{0}, b_{1}, \ldots, b_{r}$ as coefficients (predicted weights) and $x, x_{1}, \ldots, x_{r}$ as parameters, The threshold value for the logistic function output was selected by finding the maximum accuracy using the fitted model.

As an exploratory study, data were classified using two steps; an initial step to use a single parameter with a threshold for $100 \%$ specificity to exclude as many normal nodes as possible, followed by a second using a multivariate logistic model of 2 parameters to correctly classify the remaining normal nodes from metastatic nodes.

Receiver operating characteristic (ROC) analysis was used to assess the diagnostic characteristics of three individual parameters and three parameter pairs that best separate normal and metastatic nodes. This analysis was used to show the performance measurement of the logistic regression model at varying thresholds. The ROC curves illustrate trade-off between sensitivity and specificity at different thresholds by plotting sensitivity/true positive rate (TPR) against false positive rate (FPR). 

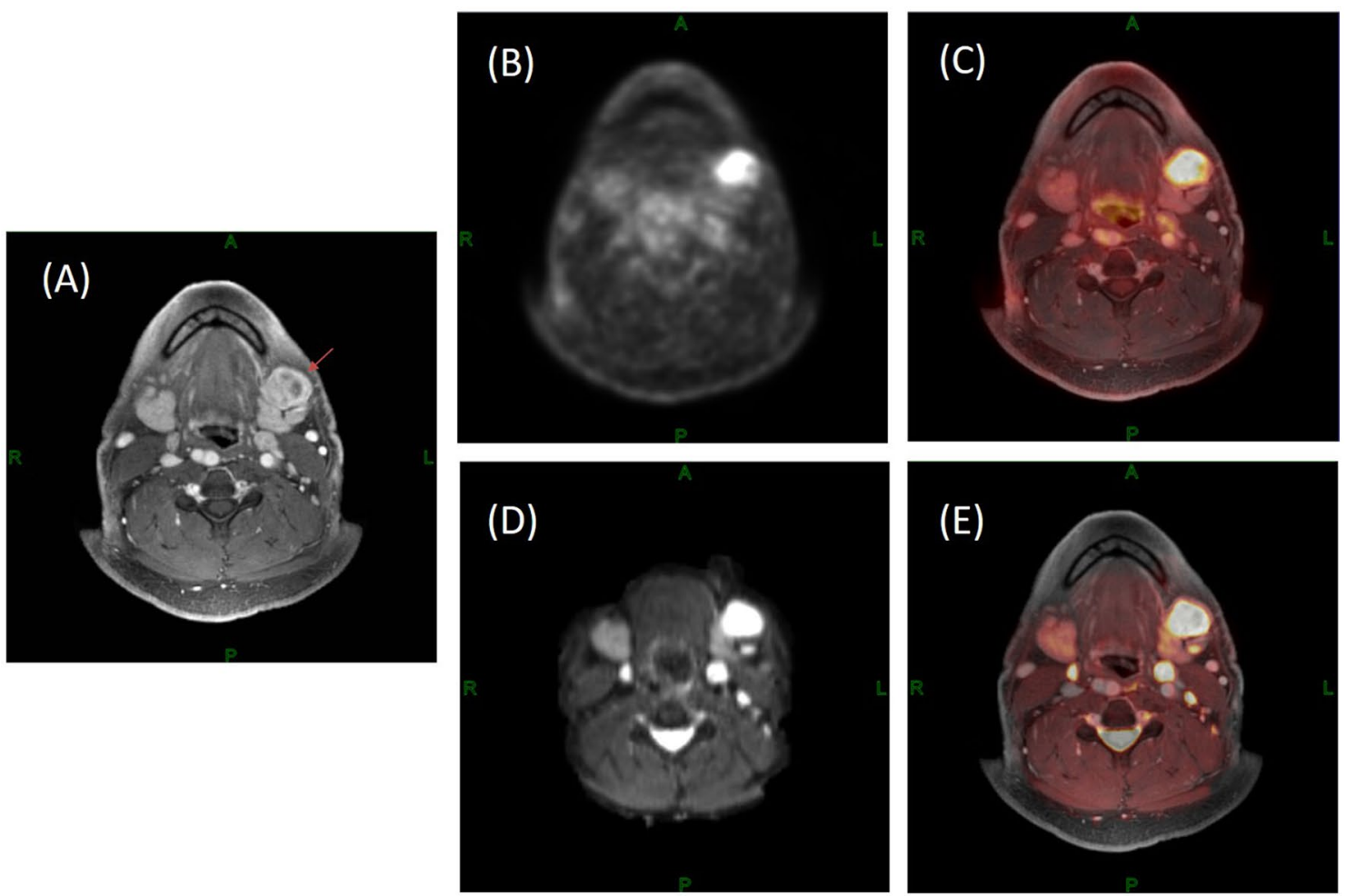

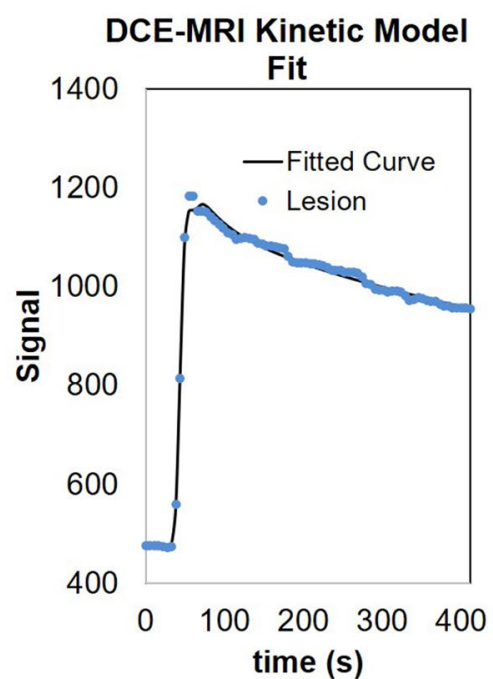

(F)

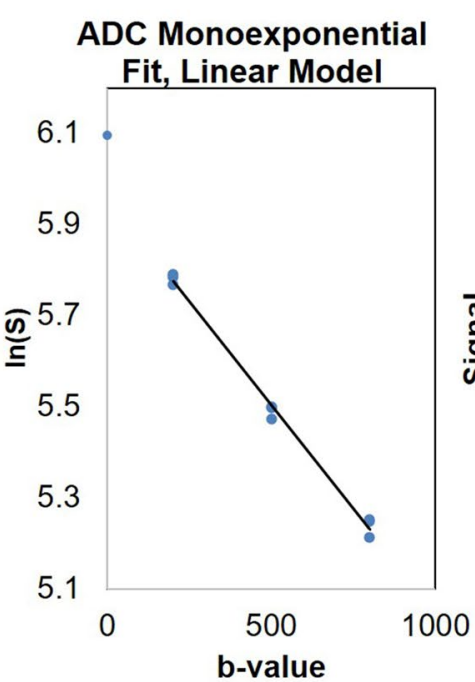

(G)
PET Kinetic Model Fit

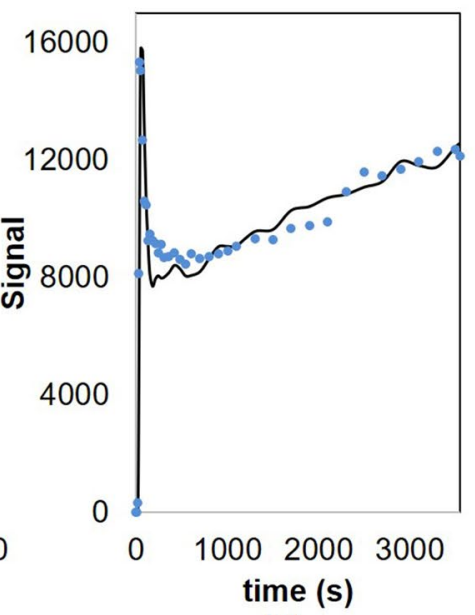

$(\mathrm{H})$

Figure 1. An example of a 50-year-old male patient with HNSCC in the floor of the mouth. A left level-1 metastatic lymph node can be detected in co-registered DCE-MRI (SimpleElastix v0.10.0; https://github.com/ SuperElastix/SimpleElastix) (A), co-registered 18F-FDG-PET image (B), PET activity map overlaid on DCEMRI (C), co-registered DWI b0 image (D) and DWI b0 image overlaid on DCE-MRI (E). The average data (blue dots) of the left level 1 metastatic lymph node (arrows) are shown for DCE-MRI (F), DWI (G), and PET (H) with their corresponding model fits (solid black lines).

\section{Results}

There were 20 HNSCC patients in this study (Table 1). On average, each patient had 3-4 levels dissected, such that there were 79 levels included in this study. All positive nodes were included for the analysis, along with one representative normal node per level. Excluding the nodes not covered in the diffusion imaging protocol with a limited number of slices, a total of 49 nodes ( 38 normal nodes and 11 metastatic nodes) were included in the quantitative analyses. Figure 1 shows a large metastatic lymph node that can be observed in post-contrast DCEMR, FDG-PET, and DW images. It demonstrates simultaneous visualization of different measures in the same location (Fig. 1C, E) after the image co-registration and using the same ROI for data analysis of the three modalities. Figure 1 also shows the mean data from a metastatic lesion ROI for three modalities, which demonstrates that the signal models used in these modalities are appropriate. The contrast kinetic model fit to the DCE-MRI 


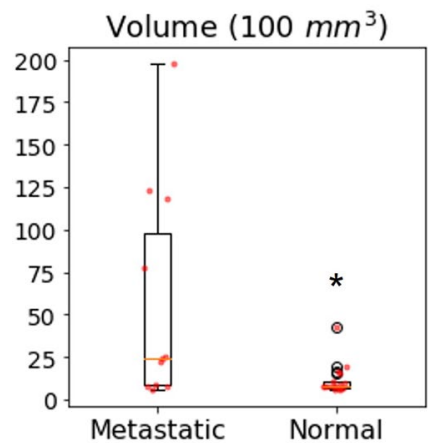

(A)

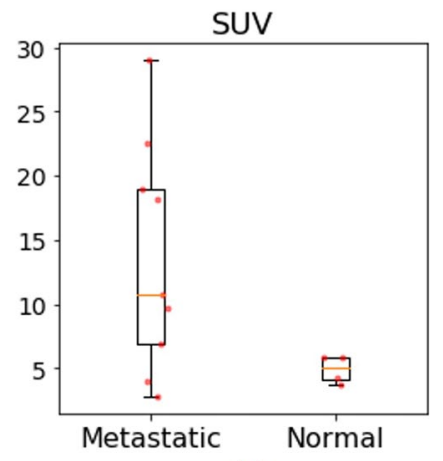

(B)

Figure 2. Box-Whisker plots of metastatic and normal lymph node volume in $100 \mathrm{~mm}^{3}$ (A) and SUV (FDGavid nodes only) (B). Red dots are for individual nodes. ${ }^{*}$ Represents a statistical significance where $p<0.05$ from Mann-Whitney U test.

data was excellent as shown in Fig. $1 \mathrm{~F}$ for both the fast wash-in and wash-out phases $\left(\mathrm{F}_{\mathrm{p}}=1.49 \times 10^{-1} \mathrm{~min}^{-1}\right.$, $\mathrm{PS}=2.64 \times 10^{-2} \mathrm{~min}^{-1}, \mathrm{~K}^{\text {trans }}=2.42 \times 10^{-2} \mathrm{~min}^{-1}, \mathrm{v}_{\mathrm{p}}=0.06$, and $\left.\mathrm{v}_{\mathrm{e}}=0.39\right)$. In DWI data's monoexponential fit (Fig. 1G), the log signal decreases linearly within the range of $b$-values used in this study $\left(\mathrm{ADC}=0.92 \mu \mathrm{m}^{2} / \mathrm{ms}\right)$. The PET data $($ Fig. $1 \mathrm{H})$ shows an initial vascular phase with a sharp peak followed by a slow irreversible uptake of tracer $\left(\mathrm{K}_{1}=1.43 \mathrm{~mL} / \mathrm{cm}^{3} / \mathrm{min}, \mathrm{k}_{2}=1.68 \mathrm{~min}^{-1}, \mathrm{k}_{3}=2.16 \times 10^{-2} \mathrm{~min}^{-1}\right.$, and $\left.\mathrm{K}_{\mathrm{i}}=1.81 \times 10^{-2} \mathrm{~mL} / \mathrm{cm}^{3} / \mathrm{min}\right)$.

Nodal size and SUV. Conventional clinical measures, nodal size and specific uptake value (SUV), are shown in Fig. 2. Node size was measured by volume $\left(\mathrm{mm}^{3}\right)$ of ROIs drawn in DCE-MRI. 34\% of normal nodes demonstrated volumes greater or equal to the smallest metastatic node. In addition, FDG-avid nodes, considered suspicious for metastasis in clinical reports, were plotted in Fig. 2B. Among the suspicious nodes, four of them were found non-cancerous in pathological evaluation and shown as normal nodes in the plot. Note that the four normal nodes have higher SUV $(5.05, \mathrm{IQR}=2.08)$ than the minimum SUV $(2.8)$ of the metastatic nodes. This shows the shortcomings of only using these clinical measures to determine the metastatic status of suspicious lymph nodes.

Quantitative MRI measures. The estimated DCE-MRI and DWI parameters are summarized in Fig. 3 and Table 2. The DCE-MRI contrast kinetic analysis results show the metastatic lymph nodes had significantly lower median $\mathrm{v}_{\mathrm{p}}($ median $=0.06(\mathrm{IQR}=0.03), p<0.01), \mathrm{F}_{\mathrm{p}}\left(7.35 \times 10^{-2}\left(1.03 \times 10^{-1}\right) \mathrm{min}^{-1}, p=0.03\right), \mathrm{PS}\left(2.64 \times 10^{-2}\right.$ $\left.\left(1.54 \times 10^{-2}\right) \mathrm{min}^{-1}, p=0.03\right)$, and $\mathrm{K}^{\text {trans }}\left(2.42 \times 10^{-2}\left(1.50 \times 10^{-2}\right) \mathrm{min}^{-1}, p=0.02\right)$ than those of the normal nodes $\left(\mathrm{v}_{\mathrm{p}}=0.08(0.01), \mathrm{F}_{\mathrm{p}}=1.42 \times 10^{-1}\left(7.07 \times 10^{-2}\right) \mathrm{min}^{-1}, \mathrm{PS}=4.24 \times 10^{-2}\left(4.93 \times 10^{-2}\right) \mathrm{min}^{-1}, \mathrm{~K}^{\text {trans }}=3.79 \times 10^{-2}\right.$ $\left.\left(3.98 \times 10^{-2}\right) \mathrm{min}^{-1}\right)$. Of the 11 metastatic nodes, compared to median $\mathrm{v}_{\mathrm{p}}$ and $\mathrm{K}^{\text {trans }}$ of normal nodes, 10 had lower $\mathrm{v}_{\mathrm{p}}$ and 8 had lower $\mathrm{K}^{\text {trans }}$, of which 8 had lower $\mathrm{v}_{\mathrm{p}}$ and $\mathrm{K}^{\text {trans. }}$. The median $\mathrm{v}_{\mathrm{e}}$ of the metastatic nodes $(0.67(0.06))$ was greater than that of normal nodes $(0.39(0.05))$ but did not reach a statistical significance. The pre-contrast T1 values for DCE-MRI data analysis were measured using either MP2RAGE or VFA method. For the cases with MP2RAGE, the median T1 values for normal and metastatic nodes were $1.30 \mathrm{~s}$ and $1.44 \mathrm{~s}$, respectively. For the cases with VFA, they were $1.40 \mathrm{~s}$ and $1.55 \mathrm{~s}$, which were not significantly different that those from MP2RAGE. There was no significant difference in ADC between the metastatic nodes $\left(0.96(0.46) \mu \mathrm{m}^{2}\right)$ and the normal nodes $\left(0.91(0.36) \mu \mathrm{m}^{2} / \mathrm{ms}\right)$.

Dynamic PET measures. Comparisons of the estimated PET pharmacokinetic parameters between metastatic and normal lymph nodes are provided in Fig. 4 and Table 2 . Metastatic nodes had significantly higher $\mathrm{k}_{3}$ $\left(1.82 \times 10^{-2}\left(1.29 \times 10^{-2}\right) \mathrm{min}^{-1}, p=8.8 \times 10^{-8}\right)$ and $\mathrm{K}_{\mathrm{i}}\left(1.23 \times 10^{-2}\left(1.11 \times 10^{-2}\right) \mathrm{mL} / \mathrm{cm}^{3} / \mathrm{min}, p=5.3 \times 10^{-8}\right) \mathrm{com}^{-}$ pared to those of normal nodes $\left(\mathrm{k}_{3}=3.02 \times 10^{-3}\left(5.57 \times 10^{-3}\right) \mathrm{min}^{-1}, \mathrm{~K}_{\mathrm{i}}=1.81 \times 10^{-3} 3.33 \times 10^{-1}\right) 5.57 \times 10^{-3} \mathrm{~mL} /$ $\left.\mathrm{cm}^{3} / \mathrm{min}\right)$. The $\mathrm{K}_{1}\left(1.20(1.77) \mathrm{mL} / \mathrm{cm}^{3} / \mathrm{min}\right)$ and $\mathrm{k}_{2}\left(1.5(1.31) \mathrm{min}^{-1}\right)$ of the metastatic nodes were also higher in metastatic nodes than they were in normal nodes $\left(\mathrm{K}_{1}=0.82(1.00) \mathrm{mL} / \mathrm{cm}^{3} / \mathrm{min}, \mathrm{k}_{2}=1.32(1.15) \mathrm{min}^{-1}\right)$, although there was no statistical significance.

Figure $4 \mathrm{E}$ shows a comparison between quantitative parameter, $\mathrm{K}_{\mathrm{i}}$, and semi-quantitative parameter, SUV, of FDG-avid and enlarged nodes that were considered suspicious for metastasis in clinical PET evaluation. There is a strong correlation $(r=0.70, p=0.008)$ between $K_{i}$ and SUV.

Classification of metastatic nodes. We investigated whether quantitative PET and MRI measures, individually or combined, can be used for classification of metastatic lymph nodes from normal ones. Using a single parameter model, $\mathrm{K}_{\mathrm{i}}$ had the highest accuracy of $96 \%$, followed by $\mathrm{k}_{3}$ for $94 \%$ and volume for $90 \%$ (Table 3 ). The ROC curves of these three parameters are shown in Fig. 5A. There were 10 different pairs of parameters for a logistic regression model that can achieve an accuracy of $96 \%$ or higher (Table 3). All 10 pairs included at least one PET parameter. Representative ROC curves of three pairs are shown in Fig. 5B. 


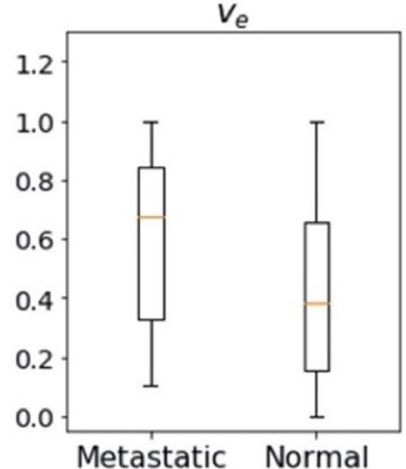

(A)

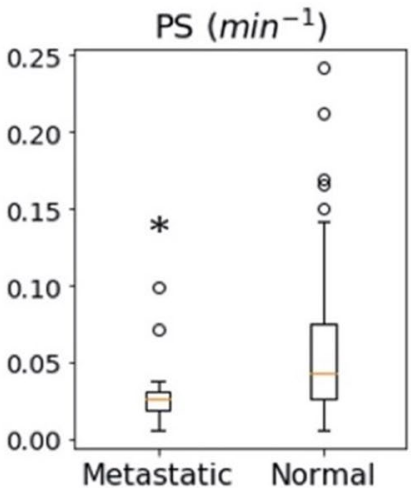

(D)

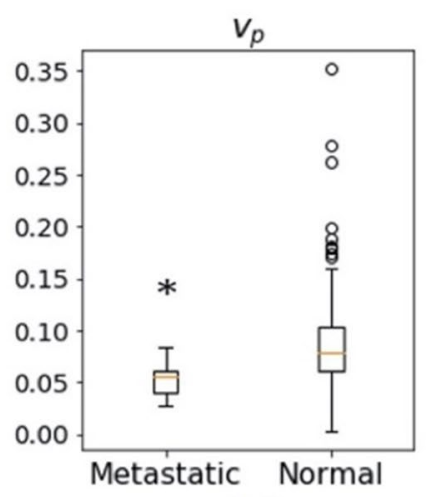

(B)

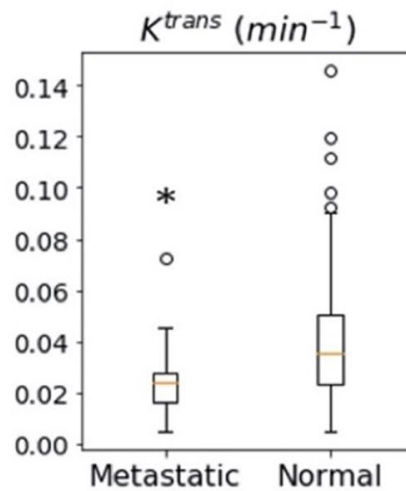

(E)

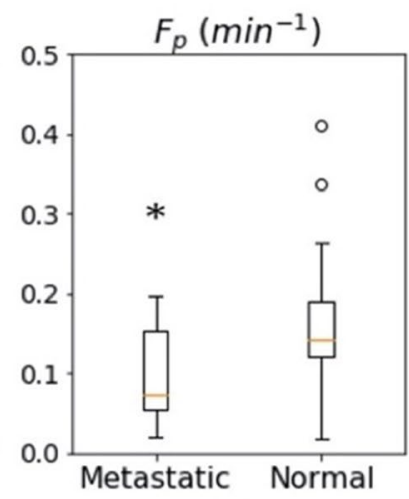

(C)

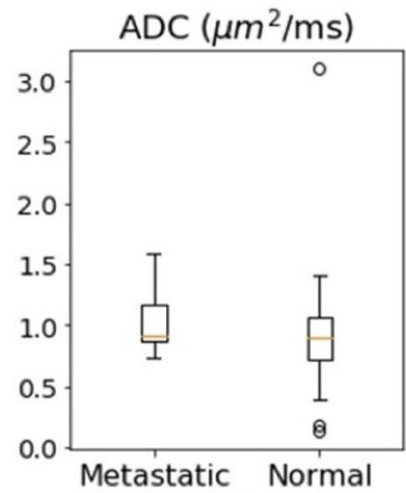

(F)

Figure 3. Comparison of DCE-MRI and DWI data between metastatic and normal nodes. Box-Whisker plots show the median and inter-quartile range of $\mathrm{v}_{\mathrm{e}}, \mathrm{v}_{\mathrm{p}}, \mathrm{F}_{\mathrm{p}}, \mathrm{PS}, \mathrm{K}^{\text {trans }}$, and ADC values of normal or metastatic nodes. ${ }^{*}$ Represents a statistical significance where $p<0.05$ from Mann-Whitney U test.

\begin{tabular}{|l|l|l|l|l|l|l|}
\hline & Normal & \multicolumn{5}{l|}{ Metastatic } \\
\cline { 2 - 7 } & Median & Q1 & Q3 & Median & Q1 & Q3 \\
\hline $\mathrm{ADC}\left(\mu \mathrm{m}^{2} / \mathrm{ms}\right)$ & 0.911 & 0.717 & 1.08 & 0.962 & 0.839 & 1.29 \\
\hline $\mathrm{v}_{\mathrm{p}}$ & $7.95 \times 10^{-2}$ & $6.08 \times 10^{-2}$ & 0.104 & $5.57 \times 10^{-2 \star}$ & $3.77 \times 10^{-2}$ & $6.41 \times 10^{-2}$ \\
\hline $\mathrm{v}_{\mathrm{e}}$ & 0.385 & 0.154 & 0.663 & 0.673 & 0.295 & 0.921 \\
\hline $\mathrm{PS}\left(\mathrm{min}^{-1}\right)$ & $4.24 \times 10^{-2}$ & $2.65 \times 10^{-2}$ & $7.58 \times 10^{-2}$ & $2.64 \times 10^{-2 \star}$ & $1.90 \times 10^{-2}$ & $3.44 \times 10^{-2}$ \\
\hline $\mathrm{F}_{\mathrm{p}}\left(\mathrm{min}^{-1}\right)$ & 0.142 & 0.120 & 0.191 & $7.35 \times 10^{-2 *}$ & $5.24 \times 10^{-2}$ & 0.156 \\
\hline $\mathrm{K}^{\text {trans }}\left(\mathrm{min}^{-1}\right)$ & $3.79 \mathrm{E}-02$ & $2.34 \times 10^{-2}$ & $6.32 \times 10^{-2}$ & $2.42 \times 10^{-2 *}$ & $1.58 \times 10^{-2}$ & $3.08 \times 10^{-2}$ \\
\hline $\mathrm{K}_{1}\left(\mathrm{~mL} / \mathrm{cm}^{3} / \mathrm{min}\right)$ & 0.816 & 0.517 & 1.52 & 1.20 & 0.657 & 2.42 \\
\hline $\mathrm{k}_{2}\left(\mathrm{~min}^{-1}\right)$ & 1.32 & 0.943 & 2.10 & 1.50 & 1.03 & 2.34 \\
\hline $\mathrm{k}_{3}\left(\mathrm{~min}^{-1}\right)$ & $3.02 \times 10^{-3}$ & $1.00 \times 10^{-4}$ & $6.04 \times 10^{-3}$ & $1.82 \times 10^{-2 *}$ & $1.05 \times 10^{-2}$ & $2.34 \times 10^{-2}$ \\
\hline $\mathrm{K}_{\mathrm{i}}\left(\mathrm{mL} / \mathrm{cm}^{3} / \mathrm{min}\right)$ & $1.81 \times 10^{-3}$ & $8.93 \times 10^{-5}$ & $3.42 \times 10^{-3}$ & $1.23 \times 10^{-2 *}$ & $7.10 \times 10^{-3}$ & $1.82 \times 10^{-2}$ \\
\hline
\end{tabular}

Table 2. Summary of the median, first quartile (Q1), and third quartile (Q3) of metastatic and normal ROI kinetic parameters. ${ }^{*} \mathrm{~A}$ statistical significance where $p<0.05$ from Mann-Whitney test.

We also investigated a two-step approach to improve the accuracy further. As described in the statistical data analysis, the first step was to use a single parameter to exclude as many normal nodes as possible. The second step was to generate a logistic regression model to classify metastatic lymph nodes from the remaining nodes. The analysis found that the best first step was to use $K_{i}$ with cutoff value of $5.3 \times 10^{-3} \mathrm{~mL} / \mathrm{cm}^{3} / \mathrm{min}$ (Fig. 6A) to remove 36 normal nodes. After this, there were only two normal nodes remaining in the pool with all metastatic nodes. There were 12 pairs of imaging parameters that were able to classify two remaining normal nodes successfully (Table 4). This second step is illustrated by two examples in Fig. 6B, C. Of the 12 two parameter combinations, volume, $\mathrm{ADC}$, and $\mathrm{F}_{\mathrm{p}}$ were the three most common parameters included and $75 \%$ of the 12 combinations included DCE-MRI parameters. 


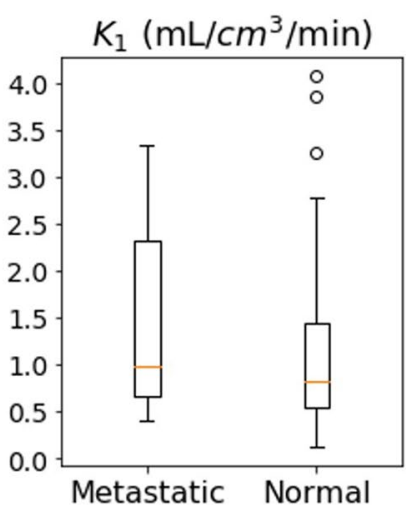

(A)

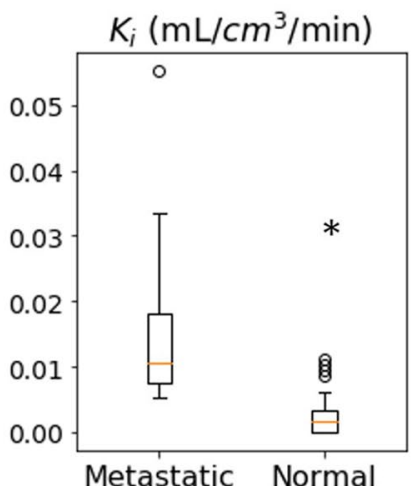

(D)

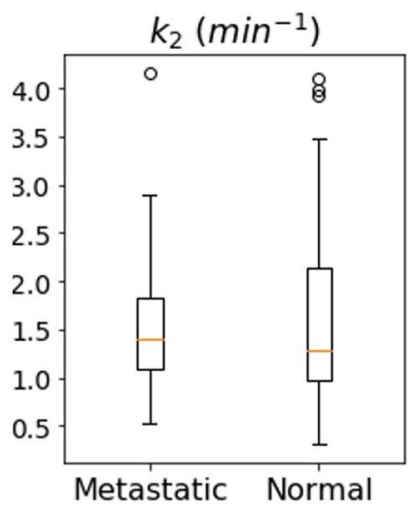

(B)

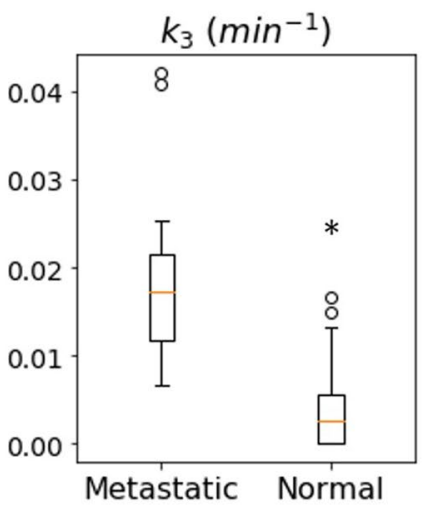

(C)

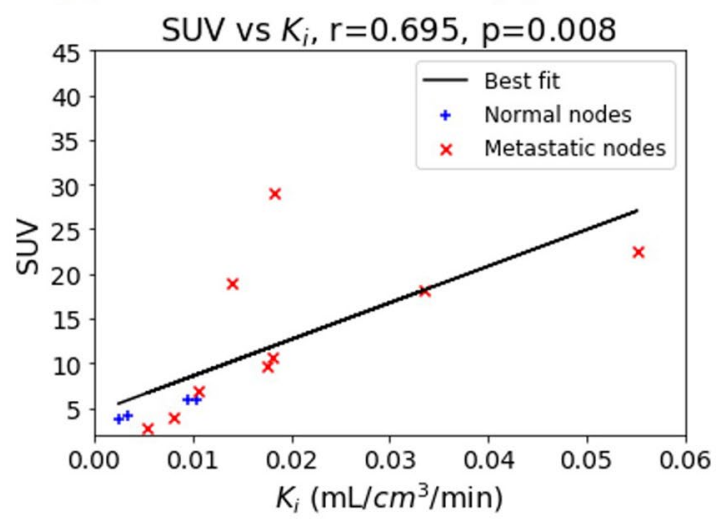

(E)

Figure 4. Comparison of dynamic PET data between metastatic and normal nodes. Box-Whisker plots show the median and inter-quartile range of $K_{1}, k_{2}, k_{3}$, and $K_{i}$ values of normal or metastatic nodes. (E) Scatter plot of $\mathrm{K}_{\mathrm{i}}$ and SUV in metastatic and normal nodes. Included in the scatter plot are the nodes that were noted as FDGavid in clinical assessment. These nodes have volumes $>574 \mathrm{~mm}^{3} .{ }^{*}$ Represents a statistical significance where $p<0.05$ from Mann-Whitney U test.

\begin{tabular}{|l|l|l|l|l|l|}
\hline Parameters & Threshold & Accuracy & Sensitivity & Specificity & AUC \\
\hline $\mathrm{K}_{\mathrm{i}}$ & 0.18 & 0.96 & 1 & 0.95 & 0.98 \\
\hline $\mathrm{k}_{3}$ & 0.43 & 0.94 & 0.82 & 0.97 & 0.97 \\
\hline Volume & 0.32 & 0.90 & 0.64 & 0.97 & 0.89 \\
\hline $\mathrm{k}_{3}, \mathrm{k}_{\mathrm{l}}$ & 0.45 & 0.98 & 0.91 & 1 & 0.99 \\
\hline $\mathrm{K}_{3}, \mathrm{k}_{2}$ & 0.36 & 0.98 & 0.91 & 1 & 0.98 \\
\hline $\mathrm{ADC}, \mathrm{K}_{\mathrm{i}}$ & 0.18 & 0.96 & 1 & 0.95 & 0.98 \\
\hline $\mathrm{K}^{\text {trans }}, \mathrm{K}_{\mathrm{i}}$ & 0.18 & 0.96 & 1 & 0.95 & 0.98 \\
\hline $\mathrm{v}_{\mathrm{e}}, \mathrm{K}_{\mathrm{i}}$ & 0.13 & 0.96 & 1 & 0.95 & 0.98 \\
\hline $\mathrm{v}_{\mathrm{e}}, \mathrm{k}_{3}$ & 0.15 & 0.96 & 1 & 0.95 & 0.99 \\
\hline $\mathrm{F}_{\mathrm{p}}, \mathrm{K}_{\mathrm{i}}$ & 0.18 & 0.96 & 1 & 0.95 & 0.98 \\
\hline $\mathrm{K}_{\mathrm{i}}, \mathrm{k}_{3}$ & 0.68 & 0.96 & 0.82 & 1 & 0.98 \\
\hline $\mathrm{K}_{\mathrm{i}}, \mathrm{k}_{2}$ & 0.18 & 0.96 & 1 & 0.95 & 0.99 \\
\hline $\mathrm{k}_{3}$, Volume & 0.54 & 0.96 & 0.82 & 1 & 0.96 \\
\hline
\end{tabular}

Table 3. Summary of parameters yielding the most accurate logistic regression prediction from all lymph nodes that overlap in DWI, DCE-MRI, and PET image field-of-view. The table includes the threshold used to achieve the highest accuracy. 


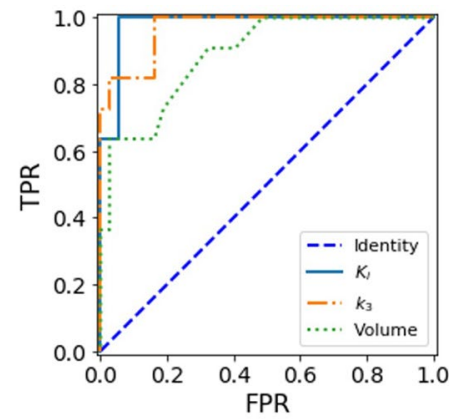

(A)

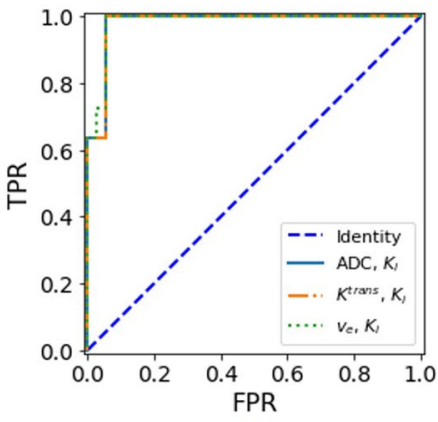

(B)

Figure 5. (A) ROC curves in single and (B) two parameter logistic regression using all 49 lymph nodes.

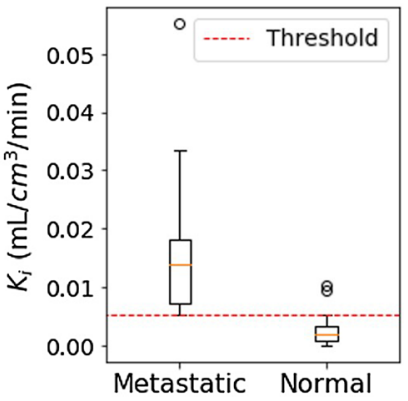

(A)

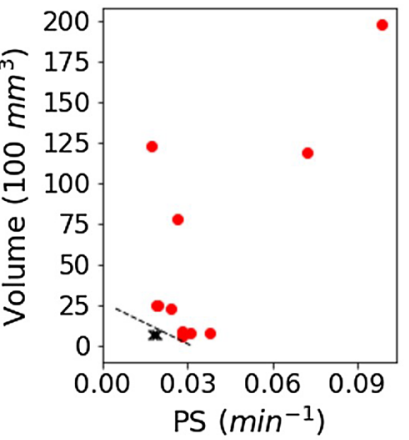

(B)

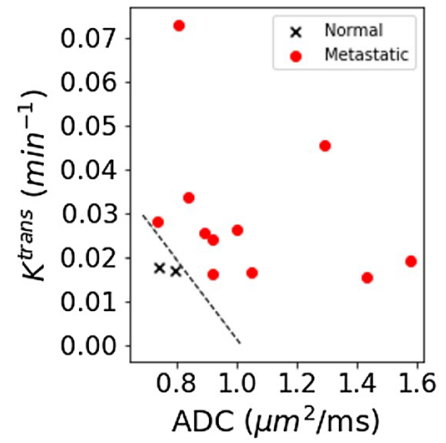

(C)

Figure 6. (A) Boxplot of $\mathrm{K}_{\mathrm{i}}$ values of lymph nodes categorized by metastatic and normal nodes showing $5.3 \times 10^{-3} \mathrm{~mL} / \mathrm{cm}_{3} / \mathrm{min}$ threshold separating metastatic from normal nodes. (B) Scatter plot of PS vs volume and (C) ADC vs Krans after removing lymph nodes under the $5.3 \times 10^{-3} \mathrm{~mL} / \mathrm{cm}^{3} / \mathrm{min}_{\mathrm{i}}$ threshold with boundary separating the remaining two normal lymph nodes from metastatic lymph nodes.

\begin{tabular}{|l|r|r|l|}
\hline Parameters $\boldsymbol{x}_{\mathbf{1}}, \boldsymbol{x}_{\mathbf{2}}$ & \multicolumn{1}{l|}{$\boldsymbol{b}_{\mathbf{1}}$} & \multicolumn{1}{l}{$\boldsymbol{b}_{\mathbf{2}}$} & Intercept \\
\hline $\mathrm{ADC}, \mathrm{PS}$ & 28.547 & 315.060 & -29.648 \\
\hline ADC, $\mathrm{K}^{\text {trans }}$ & 28.812 & 319.573 & -29.314 \\
\hline ADC, $\mathrm{F}_{\mathrm{p}}$ & 70.305 & -319.704 & -4.216 \\
\hline ADC, Volume & 119.802 & 16.013 & -208.298 \\
\hline $\mathrm{PS}, \mathrm{F}_{\mathrm{p}}$ & 384.887 & -49.744 & -0.103 \\
\hline $\mathrm{PS}$, Volume & 346.428 & 0.406 & -10.796 \\
\hline $\mathrm{K}^{\text {trans }}, \mathrm{F}_{\mathrm{p}}$ & 392.030 & -51.756 & 0.687 \\
\hline $\mathrm{K}^{\text {trans }}$, Volume & 352.125 & 0.440 & -10.587 \\
\hline $\mathrm{v}_{\mathrm{p}}$, Volume & 194.826 & 0.727 & -16.023 \\
\hline $\mathrm{F}_{\mathrm{p}}, \mathrm{k}_{2}$ & -274.030 & 41.038 & -10.704 \\
\hline $\mathrm{k}_{1}$, Volume & 18.254 & 26.594 & -209.330 \\
\hline $\mathrm{k}_{2}$, Volume & 14.005 & 10.635 & -95.187 \\
\hline
\end{tabular}

Table 4. Pairs of parameters for logistic regression models with prediction accuracy of $100 \%$ in classifying the nodes with $\mathrm{K}_{\mathrm{i}}>5.3 \times 10^{-3} \mathrm{~mL} / \mathrm{cm}^{3} / \mathrm{min}$ which was the threshold value with $100 \%$ negative predictive value to determine normal nodes. The table includes the model coefficients and intercept.

FDG-avid non-cancerous nodes. Among the four normal nodes suspected for metastasis (Fig. 4E), two of them had SUV $=5.9$ and $\mathrm{K}_{\mathrm{i}} \sim 0.01 \mathrm{~mL} / \mathrm{cm}^{3} / \mathrm{min}$. These two nodes were in level 1 and 2 of one patient as shown in Fig. 7. The subsequent surgical pathology found that these nodes had lymphoid follicular hyperplasia with numerous tingible body macrophages (Fig. 7C, D). The other two normal nodes had SUV of 3.7 and 4.2 without any particular feature to note in pathology. 


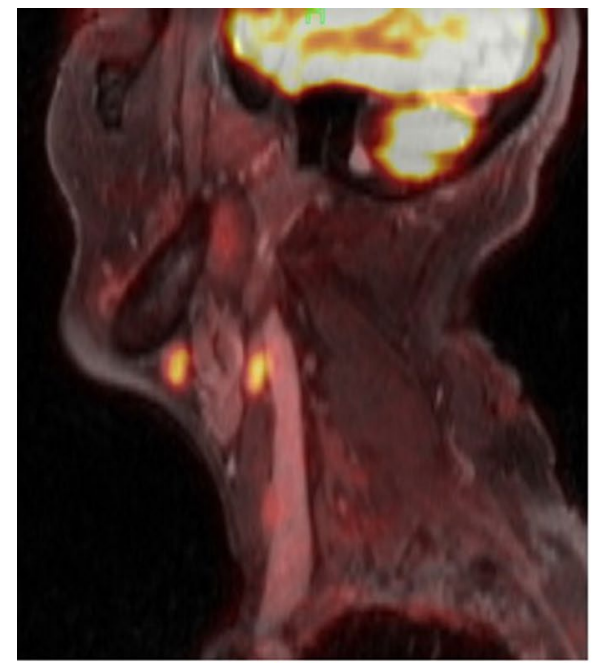

(A)

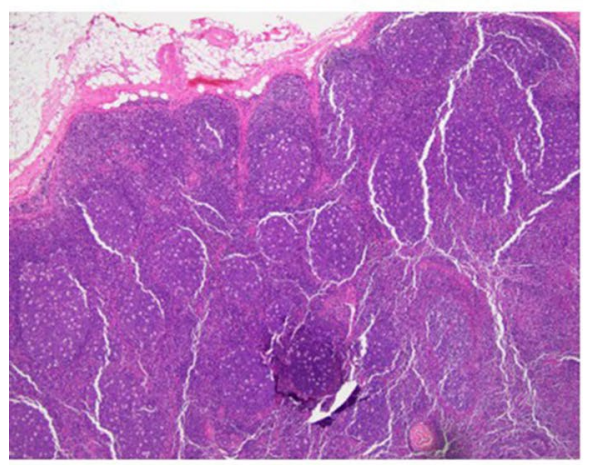

(C)

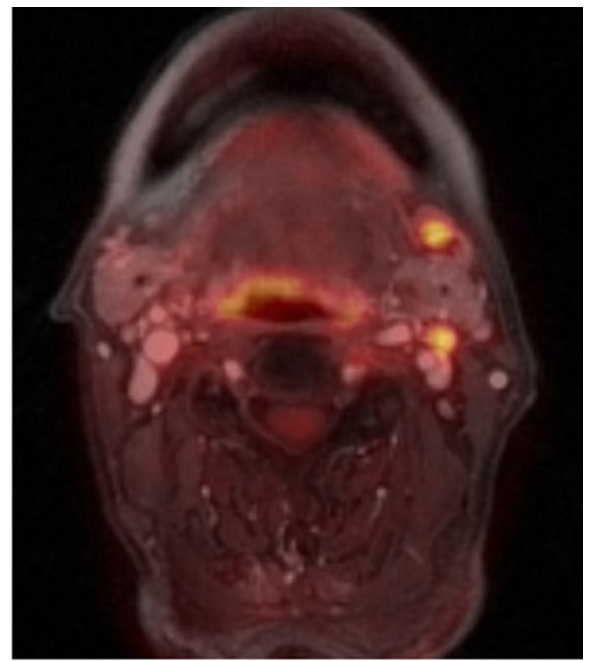

(B)

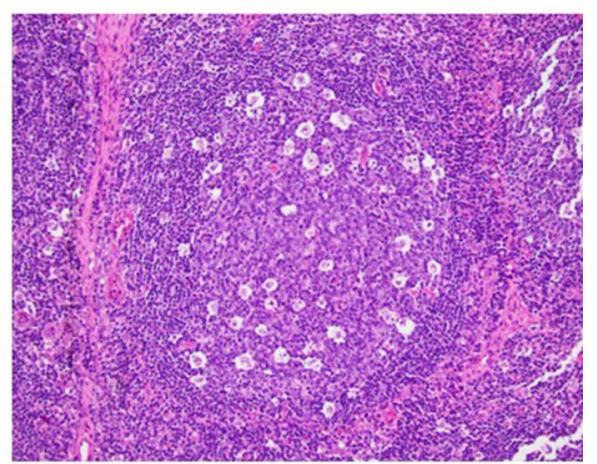

(D)

Figure 7. 93-year-old male with left mandible SCC PET activity map overlaid on co-registered DCE-MRI (SimpleElastix v0.10.0; https://github.com/SuperElastix/SimpleElastix) (top). Sagittal (A) and axial (B) slices show 2 regions with $S U V=5.9$ and mean $K_{i}=0.01 \mathrm{~mL} / \mathrm{cm}^{3} / \mathrm{min}$, discordant with negative pathology report. Low power view image showing lymphoid follicular hyperplasia $(\mathbf{C})$ in lymph node with elevated SUV and high power view image showing tingible body microphages (D).

\section{Discussion}

Quantitative parameters of DCE-MRI, FDG-PET, and DWI may have complementary diagnostic value in detecting metastatic cervical lymph nodes, in addition to using conventional clinical measures, such as volume and SUV. The results from our proof-of-concept study show the feasibility of using multiple parameters such as $\mathrm{K}_{\mathrm{i}}$, $\mathrm{ADC}$, volume, and $\mathrm{F}_{\mathrm{p}}$ to improve diagnostic accuracy in identifying metastatic lymph nodes.

Detection of metastatic lymph nodes using FDG-PET. The results of this study point to significantly higher $\mathrm{K}_{\mathrm{i}}$ value in metastatic nodes, which indicates abnormally higher metabolism compared to normal nodes. The $K_{i}$ values of the metastatic lymph nodes in our study $\left(0.016 \pm 0.014 \mathrm{~mL} / \mathrm{cm}^{3} / \mathrm{min}\right)$ were in good agreement with the values reported from a previous study with HNC patients $\left(0.023 \pm 0.004 \mathrm{~mL} / \mathrm{cm}^{3} / \mathrm{min}\right)^{40}$ and another study with a subcutaneous mouse model for non-small cell lung carcinoma $\left(0.024 \mathrm{~mL} / \mathrm{cm}^{3} / \mathrm{min}\right)^{41}$. It was noted that four metastatic nodes in our study had low $\mathrm{K}_{\mathrm{i}}$ values near the threshold and two normal nodes had $\mathrm{K}_{\mathrm{i}}>5.3 \times 10^{-3} \mathrm{~mL} / \mathrm{cm}^{3} / \mathrm{min}$. This brings up the concern of possible false positives and negatives using FDG-PET biomarkers. Previous FDG-PET studies have reported false positive findings due to inflammation in nodes ${ }^{14,42}$. Schoder et al. ${ }^{42}$ investigated radiographically negative necks (N0) using FDG-PET/CT and noted a relatively high number of false positive nodes probably from high FDG uptake due to low-level lymphadenitis caused by alcohol and tobacco smoking. Similar to our FDG-avid nodes, Schoder et al. found lymphoid follicular and parafollicular hyperplasia in their false positive cases $^{42}$. False positive nodes using PET-CT were also emphasized by Monteil et al. ${ }^{14}$, where they reported 4 of the 5 false positives were inflamed and concluded FDG-PET should be used as a guide in surgery for higher cancer stages, but not a replacement for surgery.

In addition to false positives with difficulty distinguishing inflammation from metastasis, there can also be false negative cases in smaller nodes. Yamazaki et al. ${ }^{43}$ found higher accuracy and less false positives in nodes $\geq 10 \mathrm{~mm}$ using FDG-PET compared to CT. True positive nodes had a mean diameter of $13.4 \mathrm{~mm}$ while false 
negative nodes had a mean diameter of $3.1 \mathrm{~mm}$, of which $80 \%$ were $\leq 5 \mathrm{~mm}^{43}$. Schoder et al. also pointed out that since nodes $<10 \mathrm{~mm}$ make up more than half of lymph node metastasis, PET's low spatial resolution is a limitation ${ }^{42}$. Inaccurate measures in small nodes due to low spatial resolution mentioned in these studies would lead to inaccurate parameter estimations, such as $\mathrm{K}_{\mathrm{i}}$ estimation. Thus, while FDG-PET shows good separation of metastatic and normal nodes, FDG-PET alone may not be able to differentiate these challenging cases often seen as false positives with inflammation or false negatives of small nodes. The present study results suggest that MRI measures with a higher spatial resolution can be helpful in detecting these small nodes. Further study is warranted to investigate whether there is any inherent difference among imaging modalities in terms of detecting metastatic nodes relatively smaller than their spatial resolutions.

DCE-MRI for lymph node assessment. Our data shows that metastatic nodes have significantly lower $\mathrm{v}_{\mathrm{p}}$, $\mathrm{F}_{\mathrm{p}}$, PS and $\mathrm{K}^{\text {trans }}$ than the normal nodes. This observation would not be expected in highly proliferative tumors that actively induces angiogenesis, leading to new vessel formation, and increased permeability and surface area for blood and tissue exchanges. On the other hand, such low level of vascular-related parameters could be explained by necrosis, hypoxia, and elevated interstitial fluid pressure contributing to poor perfusion, commonly observed in aggressive tumors. Previous studies have shown mixed results; significantly lower $\mathrm{K}^{\text {trans } 44}$ as well as higher $\mathrm{K}^{\text {trans45 }}$ in hypoxic nodes can be observed. Hence, it remains unclear how $\mathrm{K}^{\text {trans }}$ and other vascular-related parameters can be interpreted in terms of angiogenesis and hypoxia. Imaging hypoxia using fluoromisonidazole (FMISO) could potentially provide useful information in this regard. Further studies are warranted to improve our understanding on how these imaging parameters reflect the tumor physiological status.

The $\mathrm{K}^{\text {trans }}$ values $\left(0.0026 \pm 0.0017 \mathrm{~min}^{-1}\right)$ of metastatic and normal nodes observed in our study were relatively lower than those reported in other DCE-MRI studies of HNC that ranged from $0.189 \mathrm{~min}^{-1}$ to $0.37 \mathrm{~min}^{-146-49}$. This large discrepancy may be from using different AIF measurement methods. Our study used the populationbased AIF proposed by Parker et al. ${ }^{32}$ to avoid the influence of the variability in individual AIF measurements from partial volume effect and motion. Since this AIF was based on the measurement in the aorta and iliac arteries, its shape could have higher peak and rising rate than the measurement in a smaller peripheral vessel, resulting in lower $\mathrm{K}^{\operatorname{trans} 50}$. Hence, caution should be taken when comparing our DCE-MRI parameters with other studies. However, the comparison of these parameters between metastatic and normal nodes within our study would not be affected by the choice of AIF.

DWI for lymph node assessment. There are multiple studies that have reported significantly lower ADC in metastatic lymph nodes than that of normal nodes ${ }^{11,12,51-54}$. In the present study, however, we observed that the mean ADC in metastatic nodes was slightly greater than in normal nodes, but no significant difference was found. A similar trend was also observed by Sumi and colleagues that ADC was higher in metastatic nodes than in normal nodes ${ }^{10}$. Interestingly, the studies that reported ADC values lower in metastatic nodes than in normal nodes estimated ADC with low $b$-values including $b=0^{11,12,51-54}$. In contrary, our study and the one by Sumi et al. ${ }^{10}$ did not include any b value lower than $200 \mathrm{~s} / \mathrm{mm}^{2}$ in order to minimize the effect of intravoxel incoherent motion (IVIM) effect and found that the metastatic nodes do not have ADC lower than the normal nodes. Estimation of ADC can be influenced by many factors including IVIM, b-values, signal-to-noise ratio, and how the spatial heterogeneity of the lesions, including necrotic regions, are handled. In order to be able to combine the data from studies from multiple sites, it is imperative to have consensus on the adequate data acquisition and analysis methods specific for cancer imaging ${ }^{55}$. Future studies with such approach would enable us to establish $\mathrm{ADC}$ as a robust biomarker to assess the metastatic status of cervical lymph nodes.

Combining volume, PET, DCE-MRI, and DWI parameters for lymph node assessment. The results of our study suggest the feasibility of reducing the false-positives of FDG-PET and potentially classify lymph nodes with $100 \%$ accuracy by supplementing with MRI parameters including the nodal volume measured on MRI. Among individual parameters, $\mathrm{K}_{\mathrm{i}}$ was the best parameter to discriminate metastatic and normal nodes with $96 \%$ accuracy. However, improved accuracy could not be achieved unless a multivariate logistic regression parameter model using volume, PET, and MRI parameters was used as a second step classifier in addition to using $\mathrm{K}_{\mathrm{i}}$. Future studies with a larger cohort are required to further assess and establish this type of a multivariate diagnostic model to combine PET and MRI parameters for accurate classification of normal and metastatic.

Overall, the present study demonstrates potential value in using FDG-PET and additional MRI quantitative parameters to distinguish metastatic and normal nodes as a proof of concept study. However, there are several limitations to note. Our study took the advantage of a relatively new PET/MR scanner that can acquire both FDG-PET and MRI data simultaneously. It remains to be investigated whether similar results can be achieved using separate PET and MRI scans, despite a bigger challenge of image registration for two separate scans and the time interval between two scans with possibly different physiological conditions. Another limitation was the small cohort of patients included in this study. The result of this study suggests that the additional value of using quantitative MRI can be found with the cases with relatively low FDG uptakes. This was observed with only a handful of cases in this study. Hence, it would be crucial to recruit a larger cohort of patients for future studies. The current study was also limited to assessing the average values of the PET and MRI parameters, rather than the histogram or textual measures. There are many small nodes, particularly normal ones, which are not appropriate for histogram or textual analysis. On the other hand, there are relatively large nodes which could benefit from analyzing the spatial distribution of quantitative parameters. This histogram analysis may distinguish voxels with necrosis from other voxels in the ROI. However, it was beyond the scope of our study to investigate how to assess spatial distributions appropriately when nodes have a wide range of sizes. 


\section{Conclusion}

In this study, we assessed the feasibility of using simultaneous PET-MR imaging for assessment of metastatic status of cervical lymph nodes in HNSCC patients prior to surgery. We were able to co-register the multi-modality images and extract quantitative parameters that represent the status of nodes in terms of glucose metabolic rate, perfusion and diffusion. These parameters were successfully used to classify metastatic and normal nodes, albeit a small cohort for this proof-of-concept study. Our results suggest that quantitative MRI parameters provide additional value in distinguishing metastatic nodes, particularly among small nodes, when used together with FDG-PET. Future studies with a large cohort are warranted to further investigate the synergistic role of FDG-PET and MRI for accurate assessment of metastatic lymph nodes in head and neck cancer.

Received: 14 July 2020; Accepted: 2 November 2020

Published online: 27 November 2020

\section{References}

1. Siegel, R. L., Miller, K. D. \& Jemal, A. Cancer statistics, 2020. CA Cancer J. Clin. 70(1), 7-30 (2020).

2. de Bondt, R. B. et al. Detection of lymph node metastases in head and neck cancer: a meta-analysis comparing US, USgFNAC, CT and MR imaging. Eur. J. Radiol. 64(2), 266-272 (2007).

3. Ludwig, J. A. \& Weinstein, J. N. Biomarkers in cancer staging, prognosis and treatment selection. Nat. Rev. Cancer 5(11), 845-856 (2005).

4. Kim, S. et al. Diffusion-weighted magnetic resonance imaging for predicting and detecting early response to chemoradiation therapy of squamous cell carcinomas of the head and neck. Clin. Cancer Res. 15(3), 986-994 (2009).

5. Kim, S. et al. Prediction of response to chemoradiation therapy in squamous cell carcinomas of the head and neck using dynamic contrast-enhanced MR imaging. AJNR Am. J. Neuroradiol. 31(2), 262-268 (2010).

6. Castelijns, J. A. \& van den Brekel, M. W. Detection of lymph node metastases in the neck: Radiologic criteria. AJNR Am. J. Neuroradiol. 22(1), 3-4 (2001).

7. Heusch, P. et al. Diagnostic accuracy of ultrasound, (1)(8)F-FDG-PET/CT, and fused (1)(8)F-FDG-PET-MR images with DWI for the detection of cervical lymph node metastases of HNSCC. Clin. Oral. Investig. 18(3), 969-978 (2014).

8. Kim, S. G. et al. Potential role of PET/MRI for imaging metastatic lymph nodes in head and neck cancer. Am. J. Roentgenol. 207(2), $248-256$ (2016).

9. Torabi, M., Aquino, S. L. \& Harisinghani, M. G. Current concepts in lymph node imaging. J. Nucl. Med. 45(9), 1509-1518 (2004).

10. Sumi, M. et al. Discrimination of metastatic cervical lymph nodes with diffusion-weighted MR imaging in patients with head and neck cancer. Am. J. Neuroradiol. 24(8), 1627-1634 (2003).

11. Kim, J. K. et al. Feasibility of diffusion-weighted imaging in the differentiation of metastatic from nonmetastatic lymph nodes: Early experience. J. Magn. Reson. Imaging 28(3), 714-719 (2008).

12. Rechichi, G. et al. ADC maps in the prediction of pelvic lymph nodal metastatic regions in endometrial cancer. Eur. Radiol. 23(1), 65-74 (2013).

13. Klerkx, W. M. et al. Detection of lymph node metastases by gadolinium-enhanced magnetic resonance imaging: systematic review and meta-analysis. J. Natl. Cancer Inst. 102(4), 244-253 (2010).

14. Monteil, J. et al. Lymph node assessment with (18)F-FDG-PET and MRI in uterine cervical cancer. Anticancer Res. 31(11), 3865-3871 (2011).

15. Song, B. I. et al. 18F-FDG uptake by metastatic axillary lymph nodes on pretreatment PET/CT as a prognostic factor for recurrence in patients with invasive ductal breast cancer. J. Nucl. Med. 53(9), 1337-1344 (2012).

16. Sironi, S. et al. Lymph node metastasis in patients with clinical early-stage cervical cancer: detection with integrated FDG PET/ CT. Radiology 238(1), 272-279 (2006).

17. Nakamoto, Y. et al. Clinical value of image fusion from MR and PET in patients with head and neck cancer. Mol. Imaging Biol. 11(1), 46-53 (2009).

18. Yoon, D. Y. et al. CT, MR, US,18F-FDG PET/CT, and their combined use for the assessment of cervical lymph node metastases in squamous cell carcinoma of the head and neck. Eur. Radiol. 19(3), 634-642 (2009).

19. Som, P. M., Curtin, H. D. \& Mancuso, A. A. An imaging-based classification for the cervical nodes designed as an adjunct to recent clinically based nodal classifications. Arch. Otolaryngol. Head Neck Surg. 125(4), 388-396 (1999).

20. Comtat, C., et al. OSEM-3D reconstruction strategies for the ECAT HRRT. in IEEE Symposium Conference Record Nuclear Science 2004. 2004.

21. Marques, J. P. et al. MP2RAGE, a self bias-field corrected sequence for improved segmentation and T1-mapping at high field. Neuroimage 49(2), 1271-1281 (2010).

22. Deoni, S. C., Peters, T. M. \& Rutt, B. K. High-resolution T1 and T2 mapping of the brain in a clinically acceptable time with DESPOT1 and DESPOT2. Magn. Reson. Med. 53(1), 237-241 (2005).

23. Feng, L. et al. Golden-angle radial sparse parallel MRI: Combination of compressed sensing, parallel imaging, and golden-angle radial sampling for fast and flexible dynamic volumetric MRI. Magn. Reson. Med. 72(3), 707-717 (2014).

24. Abreu-Vieira, G. et al. Human brown adipose tissue estimated with magnetic resonance imaging undergoes changes in composition after cold exposure: An in vivo MRI study in healthy volunteers. Front. Endocrinol. (Lausanne) 10, 898 (2019).

25. Klein, S. et al. elastix: a toolbox for intensity-based medical image registration. IEEE Trans. Med. Imaging 29(1), 196-205 (2010).

26. Beare, R., Lowekamp, B. \& Yaniv, Z. Image segmentation, registration and characterization in R with SimpleITK. J. Stat. Softw. 86, 66 (2018).

27. Lowekamp, B. C. et al. The design of SimpleITK. Front. Neuroinform. 7, 45 (2013).

28. Yaniv, Z. et al. SimpleITK image-analysis notebooks: A collaborative environment for education and reproducible research. J. Digit. Imaging 31(3), 290-303 (2018).

29. Andersson, J. L., Skare, S. \& Ashburner, J. How to correct susceptibility distortions in spin-echo echo-planar images: Application to diffusion tensor imaging. Neuroimage 20(2), 870-888 (2003).

30. Smith, S. M. et al. Advances in functional and structural MR image analysis and implementation as FSL. Neuroimage 23(Suppl 1), S208-S219 (2004).

31. Andersson, J. L. R. \& Sotiropoulos, S. N. An integrated approach to correction for off-resonance effects and subject movement in diffusion MR imaging. Neuroimage 125, 1063-1078 (2016).

32. Parker, G. J. et al. Experimentally-derived functional form for a population-averaged high-temporal-resolution arterial input function for dynamic contrast-enhanced MRI. Magn. Reson. Med. 56(5), 993-1000 (2006).

33. Rata, M. et al. Assessment of repeatability and treatment response in early phase clinical trials using DCE-MRI: Comparison of parametric analysis using MR- and CT-derived arterial input functions. Eur. Radiol. 26(7), 1991-1998 (2016).

34. Lu, H. et al. Determining the longitudinal relaxation time (T1) of blood at 3.0 Tesla. Magn. Reson. Med. 52(3), 679-82 (2004). 
35. Taouli, B. et al. Hepatocellular carcinoma: Perfusion quantification with dynamic contrast-enhanced MRI. Am. J. Roentgenol. 201(4), 795-800 (2013).

36. Wake, N. et al. Accuracy and precision of quantitative DCE-MRI parameters: How should one estimate contrast concentration?. Magn. Reson. Imaging 52, 16-23 (2018).

37. Flouri, D., Lesnic, D. \& Sourbron, S. P. Fitting the two-compartment model in DCE-MRI by linear inversion. Magn. Reson. Med. 76(3), 998-1006 (2016).

38. Sokoloff, L. et al. The [14C]deoxyglucose method for the measurement of local cerebral glucose utilization: Theory, procedure, and normal values in the conscious and anesthetized albino rat. J. Neurochem. 28(5), 897-916 (1977).

39. Sanz-Requena, R. et al. Automatic individual arterial input functions calculated from PCA outperform manual and populationaveraged approaches for the pharmacokinetic modeling of DCE-MR images. J. Magn. Reson. Imaging 42(2), 477-487 (2015).

40. Lindholm, P. et al. Influence of the blood glucose concentration on FDG uptake in cancer-A PET study. J. Nucl. Med. 34(1), 1-6 (1993).

41. Yang, Z. et al. Dynamic FDG-PET imaging to differentiate malignancies from inflammation in subcutaneous and in situ mouse model for non-small cell lung carcinoma (NSCLC). PLoS ONE 10(9), e0139089 (2015).

42. Schoder, H. et al. 18F-FDG PET/CT for detecting nodal metastases in patients with oral cancer staged N0 by clinical examination and CT/MRI. J. Nucl. Med. 47(5), 755-762 (2006).

43. Yamazaki, Y. et al. Assessment of cervical lymph node metastases using FDG-PET in patients with head and neck cancer. Ann. Nucl. Med. 22(3), 177-184 (2008).

44. Jansen, J. F. et al. Noninvasive assessment of tumor microenvironment using dynamic contrast-enhanced magnetic resonance imaging and 18F-fluoromisonidazole positron emission tomography imaging in neck nodal metastases. Int. J. Radiat. Oncol. Biol. Phys. 77(5), 1403-1410 (2010).

45. Bernstein, J. M., Homer, J. J. \& West, C. M. Dynamic contrast-enhanced magnetic resonance imaging biomarkers in head and neck cancer: potential to guide treatment? A systematic review. Oral. Oncol. 50(10), 963-970 (2014).

46. Park, M. et al. Application of dynamic contrast-enhanced MRI parameters for differentiating squamous cell carcinoma and malignant lymphoma of the oropharynx. Am. J. Roentgenol. 206(2), 401-407 (2016).

47. Surov, A. et al. Correlations between DCE MRI and histopathological parameters in head and neck squamous cell carcinoma. Transl. Oncol. 10(1), 17-21 (2017).

48. Shukla-Dave, A. et al. Dynamic contrast-enhanced magnetic resonance imaging as a predictor of outcome in head-and-neck squamous cell carcinoma patients with nodal metastases. Int. J. Radiat. Oncol. Biol. Phys. 82(5), 1837-1844 (2012).

49. King, A. D. et al. DCE-MRI for pre-treatment prediction and post-treatment assessment of treatment response in sites of squamous cell carcinoma in the head and neck. PLoS ONE 10(12), e0144770 (2015).

50. Li, X. et al. Relative sensitivities of DCE-MRI pharmacokinetic parameters to arterial input function (AIF) scaling. J. Magn. Reson. 269, 104-112 (2016)

51. Abdel Razek, A. A. et al. Role of diffusion-weighted MR imaging in cervical lymphadenopathy. Eur. Radiol. 16(7), 1468-1477 (2006).

52. Holzapfel, K. et al. Value of diffusion-weighted MR imaging in the differentiation between benign and malignant cervical lymph nodes. Eur. J. Radiol. 72(3), 381-387 (2009).

53. Zhang, Y. et al. Apparent diffusion coefficient values of necrotic and solid portion of lymph nodes: Differential diagnostic value in cervical lymphadenopathy. Clin. Radiol. 68(3), 224-231 (2013).

54. Payabvash, S. Quantitative diffusion magnetic resonance imaging in head and neck tumors. Quantitative Imaging in Medicine and Surgery 8(10), 1052-1065 (2018).

55. Shukla-Dave, A. et al. Quantitative imaging biomarkers alliance (QIBA) recommendations for improved precision of DWI and DCE-MRI derived biomarkers in multicenter oncology trials. J. Magn. Reson. Imaging 49(7), e101-e121 (2019).

\section{Acknowledgements}

We are greatly indebted to the patients for participating in this study, and their physicians, A. Jacobson and T. Tran. This study was supported in part by grants from the National Institute of Health (Grant numbers: R21CA188217, R01CA160620 and UG3CA228699). The Center for Advanced Imaging Innovation and Research $\left(\mathrm{CAI}^{2} \mathrm{R}\right.$, www.cai2r.net) at New York University School of Medicine is supported by NIH/NIBIB P41 EB017183.

\section{Author contributions}

M.H. and S.G.K. designed the study. J.C., M.H., B.G., B.S., and S.G.K. conducted imaging exams and data collection. J.C., M.H., B.G., B.S., C.L., Q.C., J.L., A.M., H.R., and S.G.K. performed data analysis. J.C. prepared all figures and tables. J.C. and S.G.K. wrote the main manuscript, and all authors reviewed the manuscript.

\section{Competing interests}

The authors declare no competing interests.

\section{Additional information}

Correspondence and requests for materials should be addressed to S.G.K.

Reprints and permissions information is available at www.nature.com/reprints.

Publisher's note Springer Nature remains neutral with regard to jurisdictional claims in published maps and institutional affiliations.

Open Access This article is licensed under a Creative Commons Attribution 4.0 International format, as long as you give appropriate credit to the original author(s) and the source, provide a link to the Creative Commons licence, and indicate if changes were made. The images or other third party material in this article are included in the article's Creative Commons licence, unless indicated otherwise in a credit line to the material. If material is not included in the article's Creative Commons licence and your intended use is not permitted by statutory regulation or exceeds the permitted use, you will need to obtain permission directly from the copyright holder. To view a copy of this licence, visit http://creativecommons.org/licenses/by/4.0/.

(C) The Author(s) 2020 